\title{
Optimal Data Transmission and Channel Code Rate Allocation in Multi-path Wireless Networks
}

\author{
Keivan Ronasi*, Amir-Hamed Mohsenian-Rad ${ }^{\dagger}$, Vincent W.S. Wong*, \\ Sathish Gopalakrishnan*, and Robert Schober* \\ Department of Electrical and Computer Engineering \\ *The University of British Columbia, Vancouver, Canada \\ ${ }^{\dagger}$ Texas Tech University, Lubbock, TX \\ E-mail: *\{keivanr, vincentw, sathish, rschober $\} @$ ece.ubc.ca, ${ }^{\dagger}$ hamed.mohsenian-rad@ttu.edu
}

\begin{abstract}
Wireless links are often unreliable and prone to transmission error due to varying channel conditions. These can degrade the performance in wireless networks, particularly for applications with tight quality-of-service requirements. A common remedy is to use channel coding where the transmitter node adds redundant bits to the transmitted packets in order to reduce the error probability at the receiver. However, this perlink solution can compromise the effective link data rate, leading to undesired end-to-end performance. In this paper, we show that this latter shortcoming can be mitigated if the end-to-end transmission rates and channel code rates are selected properly over multiple routing paths. We formulate the joint channel coding and end-to-end data rate allocation problem in multipath wireless networks as a network throughput maximization problem, which is non-convex. We tackle the non-convexity by using function approximation and iterative techniques from signomial programming. Simulation results confirm that by using channel coding jointly with multi-path routing, the end-to-end network performance can be improved significantly.
\end{abstract}

\section{INTRODUCTION}

Recent advances and technological developments in communication, digital electronics, and radio frequency systems have placed wireless networks at the forefront of today's data transmission systems. Wireless links can, however, be unreliable and prone to transmission errors due to background noise, environmental obstacles, weather conditions, and user mobility. Unreliable links can degrade network performance particularly for applications with tight quality-of-service requirements such as voice-over-IP and video streaming. It is, therefore, crucial to develop efficient strategies in order to improve the reliability of data transmission in wireless networks [1].

Communication reliability in a wireless network can be improved using several methods including channel coding [2], network coding [3]-[5], data rate allocation [2], [6], and multipath routing [7]-[9]. We propose joint application of channel coding and multi-path routing to realize greater reliability when compared with using either channel coding or multipath routing on an individual basis. The central contribution of our work lies in surmounting some of the challenges posed by the optimal resource allocation problem in this setting.

Channel coding is commonly used as a tool to leverage reliable transmissions over lossy wireless links. With channel coding, the transmitter node of each link encodes the transmitted packets by adding auxiliary or redundant bits. Adding the extra bits increases the distance among the codewords and decreases the packet error probability. If the number of extra bits is the same across all wireless links, then the channel coding is non-adaptive. On the other hand, if we change the amount of redundant bits for each link based on its current channel state, then the channel coding is adaptive.

In general, channel coding introduces a trade-off between data transmission reliability and data transmission rate [2], [9][12]. In fact, by changing the code rate, i.e., the ratio of the data bits compared to data plus redundant bits, we can change the data rate at which the information is transmitted over each wireless link. In particular, the code rate can be decreased in order to improve (reduce) the probability of error at the cost of having lower data rates. Similarly, we can increase the code rate to increase the transmission data rate, but at the cost of increasing the probability of error. Through this article, we answer the following question: How should we select end-toend data transmission rates and per-link channel code rates in order to achieve the optimal rate-reliability trade-off in multipath wireless networks?

Adaptive channel coding, without multi-path routing, has been used by Lee, Chiang and Calderbank [2] to enhance the communication reliability. We introduce two key improvements to the model considered by Lee, Chiang and Calderbank. First, Lee, Chiang and Calderbank assumed that the links in the network are either wired or interference-free wireless. On the contrary, we fully incorporate the impact of wireless interference. Second, we consider the case where there are multiple end-to-end routing paths available across the network. Clearly, this includes single-path routing as a special case. These two modifications require non-trivial techniques for optimal resource allocation.

In summary, the contributions we make here are:

- We formulate network aggregate throughput maximization in multi-path wireless networks with channel coding (Section II). Both adaptive and non-adaptive channel coding are being considered.

- We tackle the non-convexity of the formulated optimization problem in two steps (Section IV). First, we introduce certain function approximations to reformulate 
the problem as a signomial programming problem. Next, we develop an iterative algorithm to solve the resulting signomial programming problem by solving a chain of tractable geometric programming problems. Although our proposed algorithm is centralized, it sheds light on the extent to which per-link channel coding can improve end-to-end performance in wireless network with multipath routing. It can also be used as a benchmark for performance comparisons with other heuristics. Further, we introduce a non-adaptive channel coding scheme with lower complexity as a sub-optimal practical solution.

- We investigate the convergence properties of the proposed algorithm as well as its efficiency. The latter is particularly studied by evaluating the impact of the approximations made in the derivation of the algorithm. In fact, we see that in spite of the approximations, the algorithm converges to the optimal solution in most cases (Section V).

- Finally, we show the advantages of an adaptive coding scheme using our design over a non-adaptive coding scheme with lower computational complexity (Section V). Through simulations, we investigate the timecomplexity tradeoff between the adaptive and nonadaptive schemes.

\section{SySTEM MODEL}

Consider an ad-hoc wireless network. We can model the network topology as a directed graph $G(\mathcal{V}, \mathcal{E})$, where $\mathcal{V}=$ $\{1,2, \ldots, V\}$ is the set of wireless nodes and $\mathcal{E}$ is the set of wireless links. Let $\mathcal{I}=\{1,2, \ldots, I\}$ denote the set of all unicast sessions in the network. For each session $i \in \mathcal{I}$, the source and destination nodes are denoted by $s_{i}$ and $t_{i}$, respectively. Furthermore, we denote $\mathcal{K}_{i}=\left\{1,2, \ldots, K_{i}\right\}$ as the set of all available routing paths from source node $s_{i}$ to destination node $t_{i}$ across the network. For each session $i \in \mathcal{I}$, each link $e \in \mathcal{E}$, and each $k \in \mathcal{K}_{i}$, we have $a_{i}^{e k}=1$ if link $e$ belongs to the $k^{\text {th }}$ routing path for session $i$, and $a_{i}^{e k}=0$ otherwise. For each session $i \in \mathcal{I}$, let $\alpha_{i}^{k}$ denote the data rate of source $s_{i}$ on its $k^{\text {th }}$ routing path, $k \in \mathcal{K}_{i}$. The aggregate transmission rate for session $i$ is obtained as $\sum_{k \in \mathcal{K}_{i}} \alpha_{i}^{k}$.

We define $R_{e}$ as the code rate of wireless link $e \in \mathcal{E}$, i.e., the ratio of the data bits to data plus redundant bits. Notice that if no channel coding is performed, then $R_{e}=1$ as there will be no redundant bits in the transmitted packet. Given the source transmission rates $\boldsymbol{\alpha}=\left(\alpha_{i}^{k}, \forall i \in \mathcal{I}, k \in \mathcal{K}_{i}\right)$ and the link code rates $\boldsymbol{R}=\left(R_{e}, \forall e \in \mathcal{E}\right)$, we can model the aggregate traffic load on wireless link $e \in \mathcal{E}$ as

$$
u_{e}=\frac{1}{R_{e}} \sum_{i \in \mathcal{I}} \sum_{k \in \mathcal{K}_{i}} a_{i}^{e k} \alpha_{i}^{k}
$$

From (1), the smaller the code rate $R_{e}$, the more redundant data is added to the transmitted packets on link $e \in \mathcal{E}$ leading to more reliable transmission (i.e., transmission with lower error probability). However, this will be at the cost of increasing the packet transmission traffic load on the link.
Let $R_{0 e} \leq 1$ denote the cut-off rate on wireless link $e \in \mathcal{E}$. We assume that the rate $R_{e}$ of the adopted coding schemes (e.g., convolutional codes) is limited by the cutoff rate [13]. Given code rate $R_{e} \leq R_{0 e}$, we can bound the packet error probability on wireless link $e$ as [13]

$$
P_{e} \leq 2^{-T\left(R_{0 e}-R_{e}\right)}
$$

where $T$ is the coding block length. In general, the cut-off rate $R_{0 e}$ depends on the signal-to-noise ratio (SNR) and the modulation scheme being used. For example, for a binary phase shift keying (BPSK) waveform [13], we have

$$
R_{0 e}=1-\log _{2}\left(1+e^{-\gamma_{e}}\right),
$$

where $\gamma_{e}$ denotes the SNR at the receiver node of wireless link $e \in \mathcal{E}$. In particular, we have

$$
\gamma_{e}=\Gamma_{e} \times d_{e}^{-\sigma} \times\left|f_{e}\right|^{2}, \quad \forall e \in \mathcal{E},
$$

where $\Gamma_{e}$ depends only on the transmission and noise powers, $d_{e}$ is the distance between the transmitter and receiver nodes of wireless link $e, \sigma$ is the path loss exponent (e.g., between 2 and 5 ), and $f_{e}$ is the small-scale fading gain.

Given the per-link failure model in (2), for each session $i \in \mathcal{I}$, the probability that a packet is transmitted successfully along the $k^{\text {th }}$ routing path, for $k \in \mathcal{K}_{i}$, is obtained as

$$
\prod_{e \in \mathcal{E}, a_{i}^{e k}=1}\left(1-P_{e}\right)=\prod_{e \in \mathcal{E}}\left(1-a_{i}^{e k} P_{e}\right) .
$$

From (5), for each session $i \in \mathcal{I}$, the aggregate receiving rate at destination node $t_{i}$ becomes

$$
\sum_{k \in \mathcal{K}_{i}} \alpha_{i}^{k} \prod_{e \in \mathcal{E}}\left(1-a_{i}^{e k} P_{e}\right) .
$$

We can model the mutual interference among the wireless links in a network by using a contention graph $G_{C}\left(\mathcal{V}_{C}, \mathcal{E}_{C}\right)$. In the contention graph $G_{C}$, the set of vertices $\mathcal{V}_{C}$ represents the set of all wireless links $\mathcal{E}$ in the network graph $G$. There exists an edge between any two vertices in set $\mathcal{V}_{C}$ if the wireless links corresponding to the two vertices mutually interfere with each other (i.e., the receiver node of one link is within the interference range of the sender node of the other link). Given the contention graph, each complete subgraph (i.e., a subgraph in which all vertices are connected to all other vertices) is called a clique. A maximal clique is then defined as a clique which is not a subgraph of any other clique [14]. We denote the set of all maximal cliques in contention graph $G_{C}$ by $\mathcal{Q}_{C}$. Only one link among all the links corresponding to the vertices of a maximal clique $Q \in \mathcal{Q}_{C}$ can be active at a time.

Let $c_{e}$ denote the nominal data rate of link $e \in \mathcal{E}$. The ratio $\frac{u_{e}}{c_{e}}$ denotes the proportion of time that link $e \in \mathcal{E}$ is active when it is being used at a data rate of $c_{e}$. It is required that

$$
\sum_{e \in Q} \frac{u_{e}}{c_{e}} \leq \nu, \quad \forall Q \in \mathcal{Q}_{C},
$$

where $\nu \in(0,1]$ is called the clique capacity. 


\section{Joint Optimal Transmission RATE AND Channel Code Rate Allocation}

Considering (1), (2), (6), and (7), the rate-reliability tradeoff can be described as follows. For each link $e \in \mathcal{E}$, by increasing the code rate $R_{e}$ we can reduce the traffic load on each link as we have already shown in (1). Thus, higher transmission rates would be allowed with the same clique capacity. On the other hand, by decreasing the code rate $R_{e}$, we can reduce the error probability in (2) which leads to higher probability of successful transmission along each routing path as shown in (5). Therefore, we may select either higher transmission rates, but with more packets being prone to error, or lower transmission rates, but with higher percentage of correctly received packets. The key question to be answered is: What transmission rates $\boldsymbol{\alpha}$ and code rates $\boldsymbol{R}$ should be selected to achieve optimal performance? To answer the above question, we formulate the optimization problem as follows:

$$
\begin{array}{cll}
\underset{\boldsymbol{\alpha} \succeq \mathbf{0}, \mathbf{0} \prec \boldsymbol{R} \preceq \boldsymbol{R}_{\mathbf{0}}}{\operatorname{maximize}} & \sum_{i \in \mathcal{I}} \sum_{k \in \mathcal{K}_{i}} \alpha_{i}^{k} \prod_{e \in \mathcal{E}}\left(1-a_{i}^{e k} P_{e}\right) & \\
\text { subject to } & P_{e} \leq 2^{-T\left(R_{0 e}-R_{e}\right)}, & \forall e \in \mathcal{E}, \\
& \sum_{e \in Q} \frac{1}{R_{e} c_{e}} \sum_{i \in \mathcal{I}} \sum_{k \in \mathcal{K}_{i}} a_{i}^{e k} \alpha_{i}^{k} \leq \nu, & \forall Q \in \mathcal{Q},
\end{array}
$$

where $\boldsymbol{R}_{\mathbf{0}}=\left(R_{0 e}, \forall e \in \mathcal{E}\right)$ denotes the vector of cut-off rates for all links in the network. The objective function in (8) is the summation of receiving rates for all sessions, where for each session $i \in \mathcal{I}$, the receiving rate is as in (6). By solving (8), we can find $\boldsymbol{\alpha}$ and $\boldsymbol{R}$ such that the total number of successfully received packets across all sessions is maximized.

\section{SOLUTION APPROACH}

In general, problem (8) is non-convex and can be difficult to solve. Note that the non-convexities in problem (8) come from the following three sources: (a) The product forms in the objective functions. (b) The exponential forms in the inequality constraints with respect to error probabilities. (c) The fractional forms in the inequality constraints with respect to clique capacities. In this section, we propose an iterative algorithm to solve the network aggregate throughput maximization problem to achieve optimal allocation of source transmission rates $\alpha$ as well as optimal channel code rates $\boldsymbol{R}$ in the network.

\section{A. Problem Reformulation to Tackle Non-convexities}

Consider the three sources of non-convexities for problem (8) which we mentioned earlier. Let us first consider the issue with having exponential forms in the inequality constraints in the error probability models. By using the Taylor series expansion, we can rewrite the error probability (2) as

$$
P_{e} \leq X_{e} \sum_{n=0}^{N_{e}} \frac{\left(L_{e} R_{e}\right)^{n}}{n !}, \quad \forall e \in \mathcal{E} .
$$

where $X_{e}=2^{-T R_{0 e}}, L_{e}=T \log 2$, and $N_{e}$ is a positive integer with $N_{e} \gg 1$. The model in (9) is in polynomial form.
Furthermore, we notice that if the error probabilities are relatively small (which is usually the case in practice), we can approximate the receiving rate for each session $i \in \mathcal{I}$ as

$$
\sum_{k \in \mathcal{K}_{i}} \alpha_{i}^{k} \prod_{e \in \mathcal{E}}\left(1-a_{i}^{e k} P_{e}\right) \approx \sum_{k \in \mathcal{K}_{i}} \alpha_{i}^{k}\left(1-\sum_{e \in \mathcal{E}} a_{i}^{e k} P_{e}\right) .
$$

Considering the worst-case packet error scenario in (9) and by replacing it, along with (10) in problem (8), we can rewrite the network aggregate throughput maximization problem as

$$
\begin{array}{ll}
\underset{\boldsymbol{\alpha}, \boldsymbol{R}}{\operatorname{maximize}} & \sum_{i \in \mathcal{I}} \sum_{k \in \mathcal{K}_{i}} \alpha_{i}^{k}\left(1-\sum_{e \in \mathcal{E}} a_{i}^{e k} X_{e} \sum_{n=0}^{N_{e}} \frac{\left(L_{e} R_{e}\right)^{n}}{n !}\right) \\
\text { subject to } & \sum_{e \in Q} \sum_{i \in \mathcal{I}} \sum_{k \in \mathcal{K}_{i}} a_{i}^{e k} \alpha_{i}^{k} R_{e}^{-1} c_{e}^{-1} \leq \nu, \quad \forall Q \in \mathcal{Q} \\
& \boldsymbol{\alpha} \succ \mathbf{0}, \mathbf{0} \prec \boldsymbol{R} \preceq \boldsymbol{R}_{\mathbf{0}} .
\end{array}
$$

The objective function and constraints in problem (11) are signomials, i.e., polynomials with both positive and negative terms. Therefore, we can apply signomial programming techniques [15] to solve problem (11) as we explain next.

Let $t$ be an auxiliary variable. Problem (11) becomes

$$
\begin{array}{ll}
\underset{t, \boldsymbol{\alpha}, \boldsymbol{R}}{\operatorname{minimize}} & t^{-1} \\
\text { subject to } & t \leq \sum_{i \in \mathcal{I}} \sum_{k \in \mathcal{K}_{i}} \alpha_{i}^{k}\left(1-\sum_{e \in \mathcal{E}} a_{i}^{e k} X_{e} \sum_{n=0}^{N_{e}} \frac{\left(L_{e} R_{e}\right)^{n}}{n !}\right) \\
& \sum_{e \in Q} \sum_{i \in \mathcal{I}} \sum_{k \in \mathcal{K}_{i}}^{e k} a_{i}^{e k} \alpha_{i}^{k} R_{e}^{-1} c_{e}^{-1} \leq \nu, \forall Q \in \mathcal{Q} \\
& t>0, \quad \boldsymbol{\alpha} \succ \mathbf{0}, \quad \mathbf{0} \prec \boldsymbol{R} \preceq \boldsymbol{R}_{\mathbf{0}} .
\end{array}
$$

We can rewrite the first constraint in (12) as

$$
t+\sum_{i \in \mathcal{I}} \sum_{k \in \mathcal{K}_{i}} \sum_{e \in \mathcal{E}} \sum_{n=0}^{N_{e}} \frac{\alpha_{i}^{k} a_{i}^{e k} X_{e}\left(L_{e} R_{e}\right)^{n}}{n !} \leq \sum_{i \in \mathcal{I}} \sum_{k \in \mathcal{K}_{i}} \alpha_{i}^{k} .
$$

Next, we follow the signomial programming techniques introduced in [15] to approximate the polynomial on the right-hand side of (13), which is a function of only $\boldsymbol{\alpha}$, as a monomial, i.e., a polynomial with only one term and positive multiplier. This approximation can be performed around some initial point $\hat{\boldsymbol{\alpha}}$. For a parameter $f_{s}>1$, which is close to 1 , we have

$$
\begin{aligned}
& \sum_{i \in \mathcal{I}} \sum_{k \in \mathcal{K}_{i}} \alpha_{i}^{k} \approx\left(\sum_{i \in \mathcal{I}} \sum_{k \in \mathcal{K}_{i}} \hat{\alpha}_{i}^{k}\right) \\
& \times \prod_{i \in \mathcal{I}, k \in \mathcal{K}_{i}}\left(\frac{\alpha_{i}^{k}}{\hat{\alpha}_{i}^{k}}\right)^{\hat{\alpha}_{i}^{k} /\left(\sum_{i^{\prime} \in \mathcal{I}, k^{\prime} \in \mathcal{K}_{i}} \hat{\alpha}_{i^{\prime}}^{k^{\prime}}\right)}, \boldsymbol{\alpha} \in\left[\hat{\boldsymbol{\alpha}} / f_{s}, f_{s} \hat{\boldsymbol{\alpha}}\right],
\end{aligned}
$$

where $\left[\hat{\boldsymbol{\alpha}} / f_{s}, f_{s} \hat{\boldsymbol{\alpha}}\right]$ is a small neighborhood around initial point $\hat{\alpha}$. For notational simplicity, we define a new term $\hat{\Lambda}$, which only depends on the initial point $\hat{\boldsymbol{\alpha}}$, as

$$
\hat{\Lambda}^{-1}=\sum_{i \in \mathcal{I}} \sum_{k \in \mathcal{K}_{i}} \hat{\alpha}_{i}^{k}
$$

From (14) and (15), inequality (13) can be approximated 
around the initial point $\hat{\boldsymbol{\alpha}}$ as

$$
\begin{aligned}
& \hat{\Lambda}\left(t+\sum_{i \in \mathcal{I}} \sum_{k \in \mathcal{K}_{i}} \sum_{e \in \mathcal{E}} \sum_{n=0}^{N_{e}} \frac{\alpha_{i}^{k} a_{i}^{e k} X_{e}\left(L_{e} R_{e}\right)^{n}}{n !}\right) \\
& \times \prod_{i \in \mathcal{I}} \prod_{k \in \mathcal{K}_{i}}\left(\frac{\alpha_{i}^{k}}{\hat{\alpha}_{i}^{k}}\right)^{-\hat{\alpha}_{i}^{k} \hat{\Lambda}} \leq 1 .
\end{aligned}
$$

The above constraint is a posynomial, i.e., a polynomial with only positive terms. Replacing (16) in (12), the network throughput maximization problem becomes

$$
\begin{array}{ll}
\underset{t, \boldsymbol{\alpha}, \boldsymbol{R}}{\operatorname{minimize}} & t^{-1} \\
\text { subject to } & \hat{\Lambda}\left(t+\sum_{i \in \mathcal{I}} \sum_{k \in \mathcal{K}_{i}} \sum_{e \in \mathcal{E}} \sum_{n=0}^{N_{e}} \frac{\alpha_{i}^{k} a_{i}^{e k} X_{e}\left(L_{e} R_{e}\right)^{n}}{n !}\right) \\
& \times \prod_{i \in \mathcal{I}} \prod_{k \in \mathcal{K}_{i}}\left(\frac{\alpha_{i}^{k}}{\hat{\alpha}_{i}^{k}}\right)^{-\hat{\alpha}_{i}^{k} \hat{\Lambda}} \leq 1 \\
& \sum_{e \in Q} \sum_{i \in \mathcal{I}} \sum_{k \in \mathcal{K}_{i}} a_{i}^{e k} \alpha_{i}^{k} R_{e}^{-1} c_{e}^{-1} \leq \nu, \forall Q \in \mathcal{Q} \\
& t>0, \hat{\boldsymbol{\alpha}} / f_{s} \preceq \boldsymbol{\alpha} \preceq f_{s} \hat{\boldsymbol{\alpha}}, \mathbf{0} \prec \boldsymbol{R} \preceq \boldsymbol{R}_{\mathbf{0}} .
\end{array}
$$

The above optimization problem is a standard geometric program, which can be easily converted into a convex problem (cf. [15], [16]). Therefore, problem (17) can be solved by the interior point method [17]. Thus, we can solve the signomial programming problem (12) by iteratively solving (17).

We are now ready to introduce Algorithm 1 for solving problem (8). Algorithm 1 starts by initializing various system parameters. The initial end-to-end transmission rates $\hat{\boldsymbol{\alpha}}$ are selected such that optimization problem (17) is feasible. Several iterations are performed, where in each iteration, we solve the geometric programming problem (17) in Line 5 by using the interior point method [17]. Given the optimal transmission rates $\boldsymbol{\alpha}_{\text {opt }}$ in each iteration, we update parameter $\hat{\Lambda}$ according to (15) and correspondingly reformulate optimization problem (17) to be solved again in the next iteration. The iterations continue until the changes in optimal objective value $t_{o p t}$ is sufficiently small compared to the previous iteration.

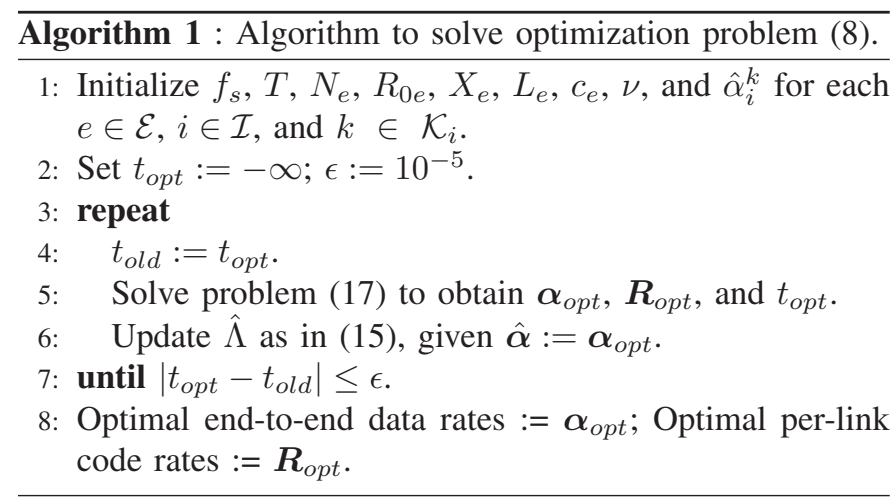

The convergence of the algorithm in each iteration is guaranteed since the interior point method is used [18]. According to [15], although there is no proof that this algorithm always converges to the optimal solution of (17), it is observed

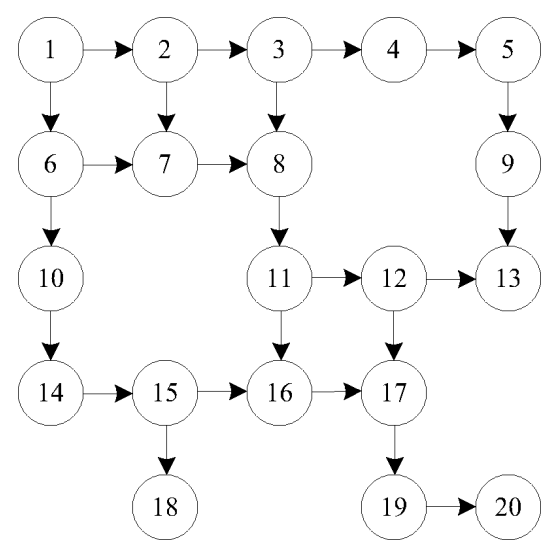

Fig. 1. A sample network with 20 nodes randomly located in a $5 \times 5$ grid and with five sessions: $1 \rightarrow 16,3 \rightarrow 13,2 \rightarrow 8,14 \rightarrow 17$, and $6 \rightarrow 20$. There are $4,2,2,1$, and 3 routing paths available for these sessions, respectively.

through simulations that Algorithm 1 always converges to the optimal solution after a number of iterations.

\section{B. Non-adaptive Channel Coding}

In this subsection, we simplify the system model in Section IV-A and assume that the channel code rate is fixed and is no longer an optimization variable in our design. That is,

$$
R_{e}=R, \quad \forall e \in \mathcal{E} .
$$

The impact of such an assumption is two-fold. First, it simplifies the clique capacity constraints in problem (8) as for each maximal clique $Q \in \mathcal{Q}$, we have

$$
\begin{aligned}
& \sum_{e \in Q} \frac{1}{R_{e} c_{e}} \sum_{i \in \mathcal{I}} \sum_{k \in \mathcal{K}_{i}} a_{i}^{e k} \alpha_{i}^{k} \\
& =\frac{1}{R}\left(\sum_{e \in Q} \sum_{i \in \mathcal{I}} \sum_{k \in \mathcal{K}_{i}} \frac{a_{i}^{e k}}{c_{e}} \alpha_{i}^{k}\right) \leq \nu
\end{aligned}
$$

This implies

$$
\sum_{e \in Q} \sum_{i \in \mathcal{I}} \sum_{k \in \mathcal{K}_{i}}\left(\frac{a_{i}^{e k}}{c_{e}}\right) \alpha_{i}^{k} \leq R \nu
$$

which is simply a linear inequality constraint. Second, since we are adding extra equality constraints to problem (8), any solution we achieve is a sub-optimal solution for problem (8).

\section{Performance Evaluation}

In this section, we assess the performance of our proposed joint channel coding and transmission data rate allocation algorithm (Algorithm 1). In our simulation model, each network topology is an $m \times m$ square grid with $V=|\mathcal{V}|=m(m-1)$ wireless nodes positioned in randomly selected grid locations. As an example, for the network in Fig. 1, we have $m=5$ and $V=20$. The network includes $m$ source and destination pairs, with potentially many available routing paths from the source node to the destination node. Unless stated otherwise, the rest of the system parameters are selected as follows: $T=10$, $N_{e}=15, f_{s}=1.1, R_{0 e}=1, \nu=\frac{2}{3}$ [19]. Without loss of generality, we choose the link capacity, $c_{e}$ for each link $e \in \mathcal{E}$, to be equal to 1 . Therefore, the transmission data rates, $\boldsymbol{\alpha}$, obtained in the optimal point can be interpreted as the vector of normalized transmission rates. We also set the initial 


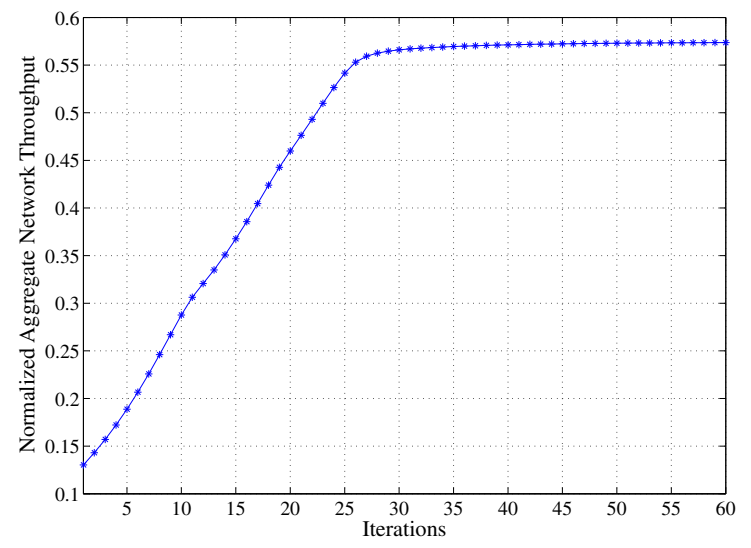

Fig. 2. Convergence of Algorithm 1 with respect to solving problem (8).

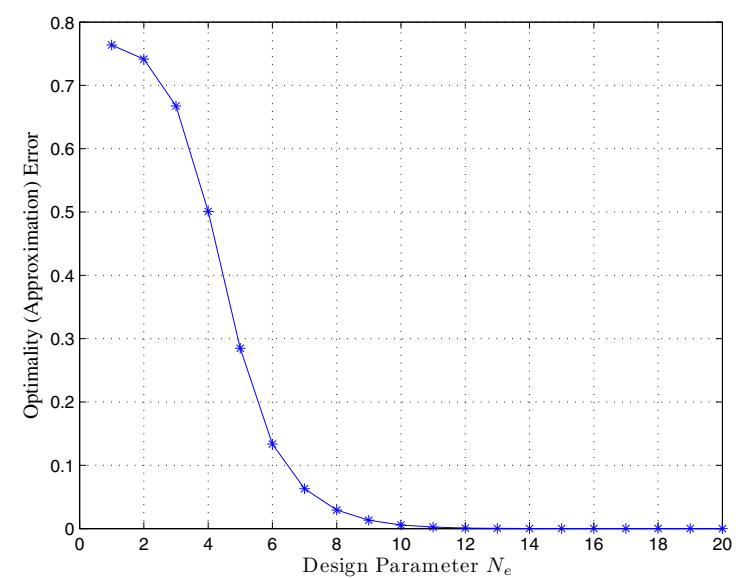

Fig. 3. The impact of parameter $N_{e}$ in approximation (9). The optimality error decreases as $N_{e}$ increases. It becomes almost zero for $N_{e}>10$.

data rates to be small, i.e., $\hat{\alpha}_{i}^{k}=0.01$ for all $i \in \mathcal{I}$ and any $k=1, \ldots, K_{i}$, in order to guarantee a feasible starting point for Algorithm 1, as we already discussed in Section IV-A.

Algorithm 1 is iterative and each iteration includes a function approximation step and a geometric programming step. Considering the topology in Fig. 1, the convergence of the objective value for problem (8), when Algorithm 1 is used, is shown in Fig. 2. Recall that the objective value for problem (8) is the network aggregate throughput. From the results in Fig. 2, Algorithm 1 converges after around 30 iterations. Similar results can be obtained for other network topologies.

Recall from Section IV that we use approximation (9) in order to convert optimization problem (8) into a tractable geometric programming problem as in (11). We can improve the accuracy of the approximation in (9) by increasing the value of $N_{e}$. However, this would be at the cost of making optimization problem (11) more complicated. Therefore, we are interested in choosing $N_{e}$ to obtain a reasonable accuracy with low computational complexity. Considering 50 random topologies, the simulation results, when $N_{e}$ varies from 1 to 20, are shown in Fig. 3, where each point indicates the average optimality error observed among all topologies. We define the optimality error as the difference between the achieved throughput at a particular choice of $N_{e}$ and that at sufficiently

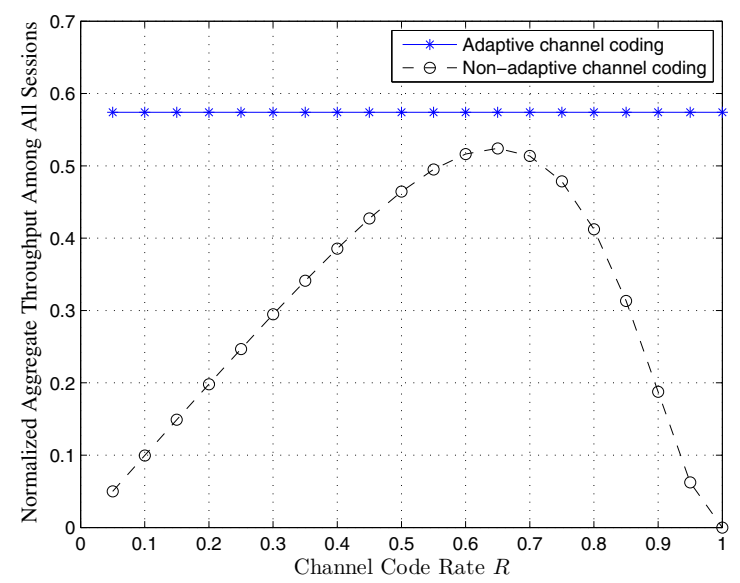

Fig. 4. Comparison between adaptive vs non-adaptive channel coding.

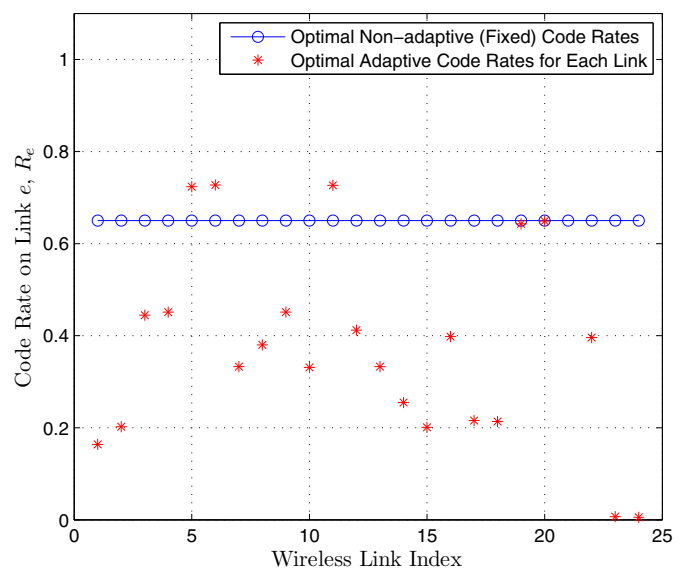

Fig. 5. Optimal adaptive versus non-adaptive code rate distribution among all links for the network in Fig. 1.

large choice of $N_{e}$, i.e., $N_{e}=20$. Results in Fig. 3 show that the optimality error approaches zero when $N_{e}$ is 10 or higher.

We now present how choosing the code rate for each link individually (i.e., adaptive channel coding) can lead to different optimality and computational complexity results, compared to the case when channel coding is non-adaptive. Recall from Section IV-B that in a non-adaptive channel coding scenario, all wireless links adopt the same code rate $R$ as expressed in (18). In this case, for each fixed $R$, optimization problem (8) becomes a linear program. As we explained in Section IV-B, this can significantly reduce the computational complexity, but results in sub-optimal design solutions. Consider the network in Fig. 1. The corresponding simulation results are shown in Fig. 4. Here, we examine various choices of the non-adaptive code rate $R$ within the feasible range $\left[0, R_{0}\right]$. We can see that by using non-adaptive channel coding, the highest throughput is achieved when the code rate on all links is 0.65 . At this point, we reach $89 \%$ of the optimal value that is achievable by using adaptive channel coding. It is interesting to also look at the distribution of the optimal adaptive code rates at all links, compared to the optimal non-adaptive code rate. In the adaptive channel coding case, the optimal code rates for various links can be significantly different. The corresponding 


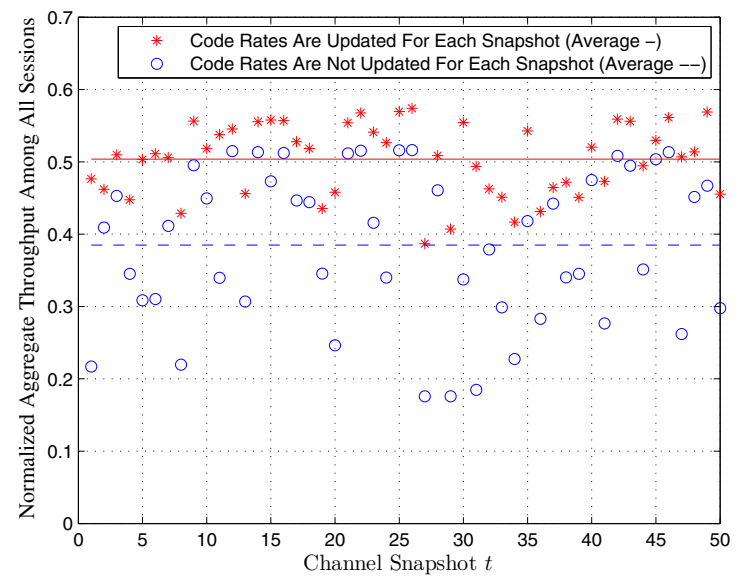

Fig. 6. Performance in a fading channel for 50 channel snapshots. We can see that it is more efficient to update the code rates at each snapshot compared to the case that code rates are obtained once for all snapshots.

results are shown in Fig. 5.

Finally, we study the impact of fading on the system performance when Algorithm 1 is used. Recall from Section IV-A that we can incorporate the impact of fading by separately solving optimization problem (8) for each wireless channel realization with fading gains $f_{e}$ and corresponding cut-off rates as in (3) and (4). In this case, Algorithm 1 is invoked every time new channel measurement data becomes available. We refer to each channel measurement data as one channel snapshot. Simulation results for the network topology in Fig. 1 for 50 different channel snapshots are shown in Fig. 6. In our simulation model, we generate the fading gains at each channel snapshot based on a random realization of the Rayleigh fading distribution. For the results in Fig. 6, we compare the performance in two design scenarios. The first design is an adaptive channel coding scheme based on the average fading information. That is, solving optimization problem (8) only once by assuming that the fading gains take their average values within the Rayleigh fading distribution. On the other hand, in our second design, we solve problem (8) once for each channel snapshot. We can see that on average, the latter case (solid line) can improve the aggregate throughput among all end-to-end sessions compared to the former one (dash line). The achieved performance improvement is at the cost of a significantly higher computational complexity due to the requirement of solving optimization problem (8) for each snapshot, which may not always be desired in practice.

\section{CONCLUSiON}

In this paper, we considered the problem of using per-link channel coding in wireless networks with multi-path routing. We focused on per-link channel code rate selection and end-toend transmission data rate allocation through network throughput maximization, which results in a non-convex problem. We tackled the non-convexity by using appropriate function approximations and iterative techniques from signomial programming. Moreover, we studied different variations of our proposed per-link channel code rate selection and end-to-end data rate allocation algorithms to address both adaptive and non-adaptive channel coding and also the impact of fading. Simulation results confirmed that by using channel coding jointly with multi-path routing, we can significantly improve the end-to-end network performance. We also showed through simulations that as a sub-optimal approach with less complexity, non-adaptive channel coding achieves a high degree of optimality compared to adaptive channel coding. As for future work, we will consider the problem of finding the optimal data transmission and channel code rates in a distributed manner.

\section{ACKNOWLEDGMENT}

This research is supported by the Natural Sciences and Engineering Research Council (NSERC) of Canada.

\section{REFERENCES}

[1] A. Beljadid, A. S. Hafid, and A. Gendreau, "Design of wireless mesh networks: Expansion and reliability studies," in Proc. of IEEE Globecom, New Orleans, LA, Dec. 2008.

[2] J. W. Lee, M. Chiang, and A. R. Calderbank, "Price-based distributed algorithms for rate-reliability based tradeoff in network utility maximization," IEEE J. Select. Areas Commun., vol. 24, no. 5, pp. 962-976, May 2006.

[3] M. Ghaderi, D. Towsley, and J. Kurose, "Reliability gain of network coding in lossy wireless networks," in Proc. of IEEE Infocom, Phoenix, AZ, Apr. 2008.

[4] D. S. Lun, M. Medard, R. Koetter, and M. Effros, "Further results on coding for reliable communication over packet networks," in Proc. of ISIT, Adelaide, Australia, Sept. 2005.

[5] K. Ronasi, A. H. Mohsenian-Rad, V. W. S. Wong, S. Gopalakrishnan, and R. Schober, "Reliability-based rate allocation in wireless intersession network coding systems," in Proc. of IEEE Globecom, Honolulu, HI, Dec. 2009.

[6] S. Fashandi, S. O. Gharan, and A. K. Khandani, "Path diversity in packet switched networks: Performance analysis and rate allocation," in Proc. of IEEE Globecom, Washington, DC, Nov. 2007.

[7] Z. Ye, S. V. Krishnamurthy, and S. K. Tripathi, "A framework for reliable routing in mobile ad hoc networks," in Proc. of IEEE Infocom, San Francisco, CA, Apr. 2003.

[8] Y. Fan, J. Zhang, and X. Shen, "Mobility-aware multi-path forwarding scheme for wireless mesh networks," in Proc. of IEEE WCNC, Las Vegas, NV, Apr. 2008.

[9] S. Dulman, T. Nieberg, J. Wu, and P. Havinga, "Trade-off between traffic overhead and reliability in multipath routing for wireless sensor networks," in Proc. of IEEE WCNC, New Orleans, LA, Mar. 2003.

[10] J. W. Lee, M. Chiang, and A. R. Calderbank, "Distributed algorithms for optimal rate-reliability tradeoff in networks," in Proc. of IEEE ISIT, Adelaide, Australia, Sept. 2005.

[11] D. O'Neill, B. S. Thian, A. Goldsmith, and S. Boyd, "Wireless NUM: Rate and reliability tradeoffs in random environments," in Proc. of IEEE WCNC, Budapest, Hungary, Apr. 2009.

[12] Y. Li, M. Chiang, R. Calderbank, and S. N. Diggavi, "Optimal ratereliability-delay tradeoff in networks with composite links," IEEE Trans. on Communications, vol. 57, no. 5, pp. 1390-1401, May 2009.

[13] J. G. Proakis, Digital Communications. 4th edition, New York: McGraw-Hill, 2001.

[14] R. Gupta and J. Walrand, "Approximating maximal cliques in ad-hoc networks," in Proc. of IEEE PIMRC, Barcelona, Spain, Sept. 2004.

[15] S. Boyd, S. J. Kim, L. Vandenberghe, and A. Hassibi, A Tutorial on Geometric Programming. Springer Science and Business Media, 2007.

[16] M. Chiang, Geometric Programming for Communication Systems. NOW, 2005.

[17] S. Boyd and L. Vandenberghe, Convex Optimization. Cambridge University Press, 2004.

[18] Y. Nesterov and A. Nemirovsky, Interior Point Polynomial Algorithms in Convex Programming. SIAM, 1994.

[19] C. Shannon, "A theorem on coloring the lines in the network," J. Math. Phys., vol. 28, pp. 148-151, Sept. 1949. 